\title{
Deficiency of macrophage migration inhibitory crossark factor attenuates tau hyperphosphorylation in mouse models of Alzheimer's disease
}

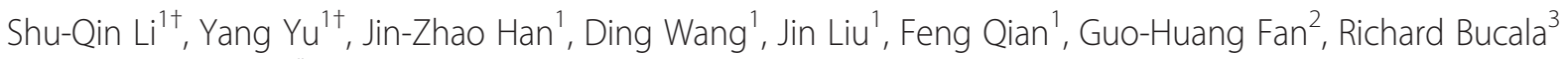
and Richard D. Ye $1 \mathrm{~A}^{1 *^{*}}$

\begin{abstract}
Background: Pathological features of Alzheimer's disease (AD) include aggregation of amyloid beta (A $\beta$ ) and tau protein. Macrophage migration inhibitory factor (MIF), a proinflammatory cytokine, has been implicated in the toxicity of aggregated A $\beta$. It remains unclear whether MIF affects hyperphosphorylation and aggregation of tau.

Methods: The effects of MIF deficiency in tau hyperphosphorylation were examined in $\mathrm{Mif}^{-{ }^{-}}$- mice receiving intracerebroventricular (ICV) injection of streptozotocin (STZ) and in APP/PS1 transgenic mice mated with Mif $^{-1-}$ mice. MIF expression and astrocyte activation were evaluated in ICV-STZ mice using immunofluorescence staining. Cultured primary astrocytes were treated with high glucose to mimic STZ function in vitro, and the condition medium (CM) was collected. The level of tau hyperphosphorylation in neurons treated with the astrocyte CM was determined using Western blotting.

Results: MIF deficiency attenuated tau hyperphosphorylation in mice. ICV injection of STZ increased astrocyte activation and MIF expression in the hippocampus. MIF deficiency attenuated astrocyte activation in ICV-STZ mice. CM from high glucose-treated WT astrocytes increased tau hyperphosphorylation in cultured primary neurons, an effect absent from $\mathrm{Mif}^{-1-}$ astrocytes and WT astrocytes treated with the MIF inhibitor ISO-1. ISO-1 had no direct effect on tau phosphorylation in cultured primary neurons.

Conclusions: These results suggest that MIF deficiency is associated with reduced astrocyte activation and tau hyperphosphorylation in the mouse AD models tested. Inhibition of MIF and MIF-induced astrocyte activation may be useful in AD prevention and therapy.
\end{abstract}

Keywords: Alzheimer's disease, Macrophage migration inhibitory factor, Tau phosphorylation, Astrocyte activation

\section{Background}

Accumulating evidence suggests that neuroinflammation contributes to tauopathy, the pathological accumulation of tau proteins in neurodegenerative disorders such as Alzheimer's disease (AD) [1]. The brain cells involved in AD-related neuroinflammation include microglia and astrocytes. Astrocytes, the largest and most abundant neuroglial cells in the brain, become activated in $\mathrm{AD}$ and contribute to a reinforced inflammatory cascade [2]. A variety

\footnotetext{
* Correspondence: yedequan@sjtu.edu.cn

${ }^{\dagger}$ Equal contributors

'School of Pharmacy, Shanghai Jiao Tong University, Shanghai 200240, China ${ }^{4}$ Institute of Chinese Medical Sciences, University of Macau, Macau, SAR, China

Full list of author information is available at the end of the article
}

of factors, including amyloid beta $(\mathrm{A} \beta)$ and proinflammatory cytokines, may activate astrocytes [2]. Reactive astrocytes in turn produce nitric oxide and proinflammatory cytokines such as interleukin-1 (IL-1) and IL-6, which accelerate tauopathy including formation of neurofibrillary tangles (NFTs) [3-5]. Although these findings suggest a possible link between neuroinflammation and tauopathy, there is little evidence for a direct role of reactive astrocytes in the pathological accumulation of the microtubule-associated protein tau.

Macrophage migration inhibitory factor (MIF) is a proinflammatory cytokine found in many tissues and in cells such as monocytes and macrophages [6]. MIF has multiple functions including catalytic activities [7], lymphocyte 
activation [8], glucocorticoid counter-modulation [9], and proinflammatory regulation [10]. In addition to modulating the immune system, MIF is involved in tumorigenesis [11]. However, the function of MIF in the brain is not well understood. MIF is found in neurons, astrocytes, and microglia of the hippocampus and cortex [12], and proinflammatory stimuli significantly upregulate the expression of neuronal MIF at both mRNA and protein levels [13]. Clinical studies have shown a marked increase in MIF concentration in the cerebral spinal fluid (CSF) of AD patients [14]. Another piece of evidence indicating MIF involvement in $\mathrm{AD}$ is the elevated expression of CD74, which is a receptor for MIF [12, 15]. MIF has also been functionally linked to the toxicity of $\mathrm{A} \beta$ aggregates, which is a major constituent of AD plaques [16, 17]. Despite these findings, a correlation between MIF and tauopathy has not been established experimentally.

Tau hyperphosphorylation is a major pathological feature of neurodegenerative disorders such as AD [18]. In this study, we evaluated the effects of MIF deficiency in tau hyperphosphorylation using two mouse models of $\mathrm{AD}$, one involving intracerebroventricular (ICV) injection of streptozotocin (2-deoxy-2-(3-methyl-3-nitrosoureido)-D-glucopyranose; STZ) into WT and $\mathrm{Mif}^{-1-}$ mice, and the other being the APP/PS1 transgenic mice mated with $\mathrm{Mif}^{-1-}$ mice. The results have shown that MIF deficiency significantly attenuated tau hyperphosphorylation in these mouse models. Moreover, the expression of MIF in astrocytes and the number of reactive astrocytes were markedly increased in the AD models, whereas MIF deficiency is associated with decreased astrocyte activation in these mice. Finally, we found that conditioned medium from activated astrocytes could stimulate tau hyperphosphorylation in neurons in a MIF-dependent manner. Based on these findings, we postulate that MIF plays a role in tauopathy through activation of astrocytes.

\section{Methods}

\section{Reagents}

Primary antibodies used in this study are listed in Table 1. Dulbecco's modified Eagle's medium (DMEM), neurobasal-A and B-27 ${ }^{\circ}$ supplements were purchased from Gibco (Invitrogen, Carlsbad, CA). The BCA protein assay kit and 4,6-diamidino-2-phenylindole (DAPI) were obtained from Beyotime Institute of Biotechnology (Nantong, Jiangsu, China). Mouse and rabbit control IgGs were purchased from Santa Cruz Biotechnology (Dallas, TX). IRDye $800 \mathrm{CW}$ secondary antibodies were from LICOR (Lincoln, NE). Other chemicals were obtained from Sigma Chemical Company (St. Louis, MO).

\section{Mice and treatments}

The Mif $^{-1}$ (MIF-KO) mice in C57BL/6 background were prepared as reported previously [19]. All mice were housed (four to five animals per cage) with a 12/ $12 \mathrm{~h}$ light/dark cycle, with ad libitum access to food and water. The housing, breeding, and animal experiments were in accordance with the National Institutes of Health Guide for the Care and Use of Laboratory Animals, with procedures approved by the Biological Research Ethics Committee of Shanghai Jiao Tong University. Breeding with the APP/PS1 mice were detailed in Additional file 1.

Female WT and $\mathrm{Mif}^{-1-}$ mice of 3-6 months in age were randomly divided into control and ICV-STZ (streptozotocin, 2-deoxy-2-(3-methyl-3-nitrosoureido)-D-glucopyranose; Sigma) groups. The ICV-STZ mice were established by intracerebroventricular injection of a single dose of STZ [20]. Briefly, mice were anesthetized by intraperitoneal injection of $5 \mathrm{mg} / \mathrm{ml}$ pentobarbital sodium (Sigma) and then restrained onto a stereotaxic apparatus. Each mouse received a single ICV injection of $3 \mathrm{mg}$ STZ/kg body weight, which was freshly prepared in normal saline, into both lateral brain ventricles. The bregma coordinates used for injection were $-1.0 \mathrm{~mm}$ lateral, $-0.3 \mathrm{~mm}$ posterior, and $-2.5 \mathrm{~mm}$ below.

Table 1 Primary antibodies used in this study

\begin{tabular}{|c|c|c|c|c|}
\hline Antibody & Type & Specificity & Phosphorylation sites & Reference/source \\
\hline Tau5 & Mono- & Tau (total) & & Invitrogen, Carlsbad, CA, USA \\
\hline Tau1 & Mono- & Tau (non-phos.) & & Millipore, Temecula, CA, USA \\
\hline pS199 & Poly- & P-tau & Ser199 & Invitrogen \\
\hline pT205 & Poly- & P-tau & Thr205 & Invitrogen \\
\hline pS396 & Poly- & P-tau & Ser396 & Invitrogen \\
\hline pS404 & Poly- & P-tau & Ser404 & Invitrogen \\
\hline GFAP & Poly- & GFAP & & Proteintech, Chicago, IL, USA \\
\hline $\mathrm{GFAP}-\mathrm{Cy} 3^{\mathrm{TM}}$ & Mono- & GFAP & & Sigma-Aldrich, St Louis, MO, USA \\
\hline MIF & Poly- & MIF & & Abcam, Cambridge, MA, USA \\
\hline Anti- $\beta$-actin & Mono- & $\beta$-actin & & Sigma \\
\hline Anti-GAPDH & Poly- & GAPDH & & GoodHere Technology, Hangzhou, China \\
\hline
\end{tabular}


As controls, ICV-saline mice received an equal volume of normal saline. All mice were placed on heating pads $\left(37{ }^{\circ} \mathrm{C}\right)$ until recovered from surgery. The body weight of these mice was measured once every 7 days. Six weeks after ICV injection, all mice were sacrificed by decapitation and their brains removed immediately (Fig. 1a). The hippocampi and cerebral cortices of the mice were dissected, flash frozen in dry ice, and stored at $-80{ }^{\circ} \mathrm{C}$ for biochemical analyses later. Some brain tissues were fixed with $4 \%$ paraformaldehyde in $0.1 \mathrm{M}$ PBS, followed by cryoprotection in $30 \%$ sucrose. Sagittal sections of $30-\mu \mathrm{m}$ thickness were cut using a freezing sliding microtome. The sections were stored in glycol anti-freeze solution (ethylene glycol, glycerol, and $0.1 \mathrm{M}$ PBS in 3:3:4 ratio) at $-20{ }^{\circ} \mathrm{C}$ until immunohistochemical staining.

\section{Western blot}

Mouse brain tissue was homogenized in lysis buffer containing $50 \mathrm{mM}$ Tris- $\mathrm{HCl}$ (pH 7.4), $100 \mathrm{mM} \mathrm{NaF}, 2 \mathrm{mM}$ EDTA, $10 \mathrm{mM} \beta$-mercaptoethanol, $2 \mathrm{mM}$ sodium vanadate, $8.5 \%$ sucrose, $5 \mu \mathrm{g} / \mathrm{ml}$ aprotinin, $100 \mu \mathrm{g} / \mathrm{ml}$ leupeptin, and $5 \mu \mathrm{g} / \mathrm{ml}$ pepstatin. Protein concentrations were determined by using a BCA Kit according to the manufacturer's protocol. Tissue homogenates were separated on $10 \%$ SDS-PAGE, and separated samples were transferred onto nitrocellulose membranes (Whatman Protran ${ }^{\circ}$ ). The blots were incubated with primary antibodies and further treated with corresponding secondary antibodies before scanning using an Odyssey P140-CLx Infrared Imager (LI-COR Biosciences, Lincoln, Nebraska). Densitometric quantification of protein bands in Western blots was analyzed using the TINA software (Raytest Isotopenmessgeraete $\mathrm{GmbH}$, Straubenhardt, Germany).

\section{Immunofluorescence staining}

Sections of mouse brain were permeabilized in $3 \%$ $\mathrm{H}_{2} \mathrm{O}_{2}$ and $50 \%$ methanol in $0.05 \mathrm{M}$ TBS (containing $0.05 \mathrm{M}$ Tris buffer and $9 \mathrm{~g} / \mathrm{l} \mathrm{NaCl}$ ) for $30 \mathrm{~min}$ at room temperature, followed by blocked with blocking solution containing $5 \%$ normal goat serum and $0.1 \%$ Tween-20 in Tris-buffered saline, pH 7.4 (TBS) at room temperature for $30 \mathrm{~min}$. Sections were then incubated with primary antibodies overnight at $4{ }^{\circ} \mathrm{C}$, rinsed with TBS, and further treated with secondary antibodies for $1 \mathrm{~h}$ in the dark at room temperature. Again after three washes in TBS, sections were stained for nuclei with $5 \mu \mathrm{g} / \mathrm{ml}$ DAPI for $10 \mathrm{~min}$ at room temperature, and then mounted on glass slides. Fluorescent images were taken on a laser-scanning confocal fluorescent microscope (Leica TCS SP8, Leica Microsystems, Wetzlar, Germany). The immunofluorescence intensity was quantified using ImagePro Plus Software (Media Cybernetics, Silver Spring, MD). The results were expressed as mean \pm SEM based on a minimum of five individual fields. Student's $t$ test was used for statistical analysis with the Prism software (Ver. 5, GraphPad, San Diego, CA).

\section{Primary neuronal cultures}

Neuronal cultures were established in cortices obtained from 1-day-old $\mathrm{Mif}^{-1-}$ or WT mice as described before [21] with minor modification. In brief, cerebral cortices

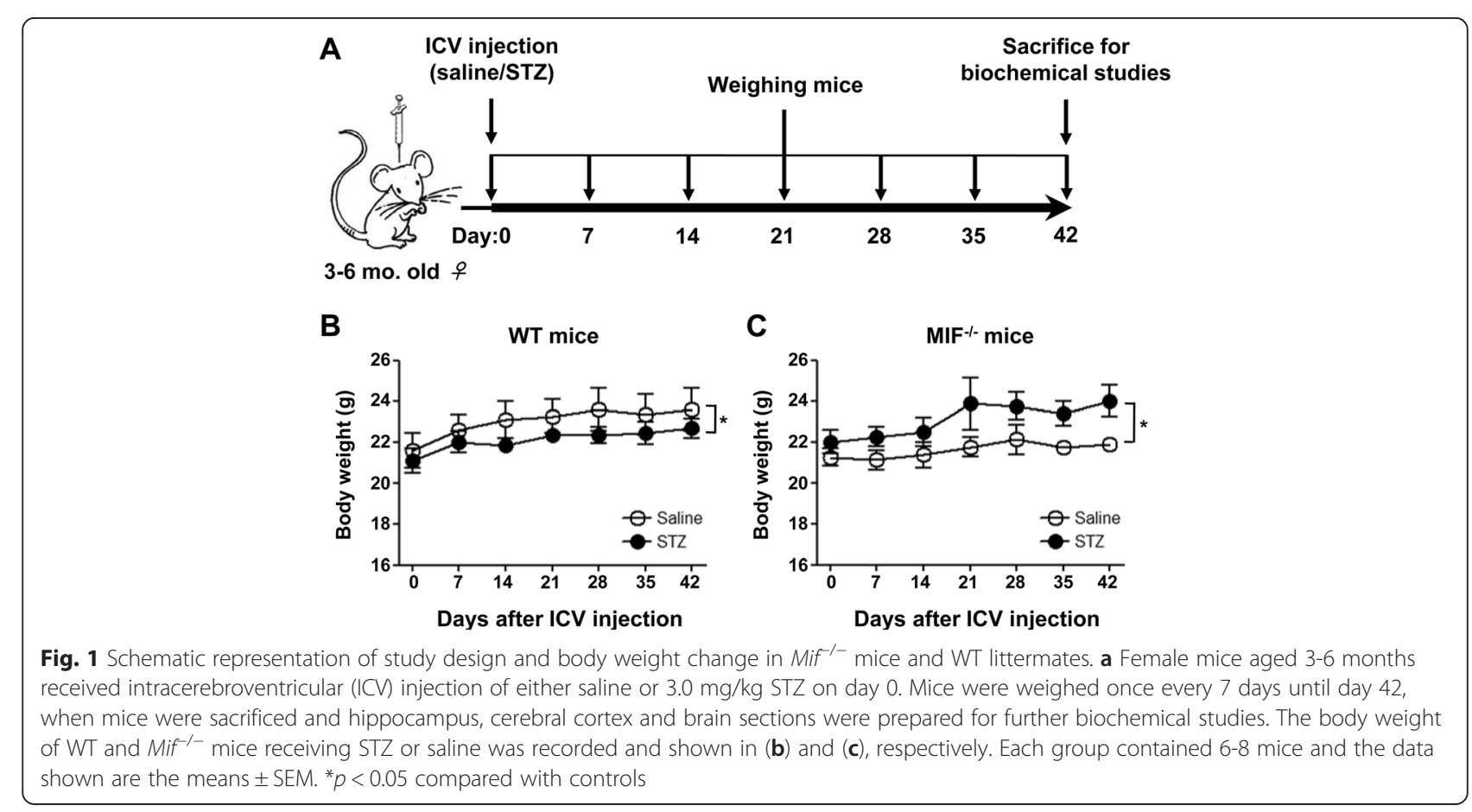


were removed from the brains of mouse pups, the meanings and microvessels were removed, and tissues were minced with a sterile razor blade. Tissues were digested with $0.025 \%$ trypsin (Sigma) and $0.01 \%$ DNase I (Sigma) at $37{ }^{\circ} \mathrm{C}$ for $10 \mathrm{~min}$. The cell suspension was filtered through a 200 mesh sieve, and cells were plated on poly-D-lysine (Sigma)-coated 24-well plates at a density of $5 \times 10^{5}$ cells per well. Two hours later, the DMEM medium (containing 10 \% FBS, $100 \mathrm{U} /$ $\mathrm{ml}$ of penicillin, and $100 \mathrm{~g} / \mathrm{ml}$ streptomycin sulfate) were replaced with neurobasal medium containing $2 \% \mathrm{~B}-27^{\circ}$ supplements for 2 days. Culture medium were changed to neurobasal with $10 \% \mathrm{FBS}$ and $3 \mu \mathrm{g} / \mathrm{ml}$ cytosine- $\beta$ $\mathrm{D}$-arabinofuranoside (Ara-C, Sigma) in the following 2 days, and then again switched back to neurobasal medium containing $2 \%$ B- $27^{\circ}$ supplements. Experiments were performed on days 7-8 after initiation of the culture.

\section{Primary astrocyte culture}

Astrocyte cultures were prepared from 1-day-old $\mathrm{Mif}^{1-}$ and WT mice as described before [22, 23]. Specifically, separated cells were cultured in poly-D-lysine-coated 75- $\mathrm{cm}^{2}$ flasks with DMEM medium (containing $10 \%$ FBS, $100 \mathrm{U} / \mathrm{ml}$ penicillin, and $100 \mathrm{~g} / \mathrm{ml}$ streptomycin sulfate). The medium was replenished on day 1 and day 3. On day 7 , microglia cells in the culture flasks were shaken off at $260 \times \mathrm{rpm}$ for $2.5 \mathrm{~h}$, and the remaining astrocytes were maintained in DMEM with $10 \%$ FBS until seeding into 6-well plates. Experiments were performed after one passage of the cells.

\section{Treatment with conditioned medium of astrocytes}

When astrocytes cultured in plates grew to $70-80 \%$ confluence, the culture medium was replaced with fresh DMEM without FBS, or with the above medium containing $75 \mathrm{mM}$ or $150 \mathrm{mM}$ glucose. The cells were incubated for another 12, 24, 48, and $72 \mathrm{~h}$. At the end of the incubation, conditioned medium $(\mathrm{CM})$ was collected and cells were lysed in $2 \times$ SDS buffer for immunoblotting analysis. The astrocyte CM collected at different time points was added to the culture of neurons from $\mathrm{Mif}^{\prime-}$ or WT mice for another 12, 24, and $48 \mathrm{~h}$. The neuronal cell lysate was prepared and analyzed by SDS-PAGE and Western blotting as detailed above.

\section{Statistical analyses}

Data are presented as means \pm SEM from at least three experiments. One-way ANOVA followed by NewmanKeuls test or Student's $t$ test was performed using the statistic software Prism 5 (GraphPad). $p<0.05$ was considered statistically significant.

\section{Results}

MIF deficiency reverses body weight decrease in ICV-STZ mice

In this study, we used two mouse models of $\mathrm{AD}$ to investigate the effect of MIF on tau phosphorylation. The first model was generated by ICV injection of STZ (ICV-STZ) $[24,25]$ in $\mathrm{Mif}^{-1-}$ mice and WT littermates, using normal saline as a control (ICV-saline). As STZ-induced animal models are characterized by insulin deficiency, elevated glucose level, and lower body weight, changes in the body weight were monitored in these mice over a period of 42 days. Mice receiving ICV-STZ had lower body weight compared with mice receiving saline (Fig. 1b). This result was consistent with a previous report using rats [26]. In the $\mathrm{Mif}^{-1}$ group, mice receiving ICV-STZ had higher body weight than those receiving ICV-saline (Fig. 1c). These results show that Mif deficiency reversed the decrease in body weight seen in ICV-STZ mice.

\section{MIF deficiency attenuates tau hyperphosphorylation in mice receiving ICV-STZ}

To investigate whether MIF affects tau phosphorylation, we examined the levels of total tau (using the Tau 5 antibody), non-phosphorylated tau (using the Tau1 antibody), and phosphorylated tau at several AD-related amino acid positions including Ser199, Thr205, Ser396, and Ser404 in the hippocampus and cerebral cortex of mice receiving ICV injection of STZ. As expected, in the hippocampi of WT ICV-STZ mice, there was an increase in tau phosphorylation at Ser199, Thr205, and Ser396 (Fig. 2a, c) when compared with the ICV-saline controls. The same comparison also identified a marked decrease in the level of non-phosphorylated tau in WT ICV-STZ mice (Fig. 2a, c). When normalized against total tau protein (Tau5), an increase in tau phosohorylation at Thr205 and Ser396 was evident (Fig. 2b). In the $\mathrm{Mif}^{/-}$mice, however, no significant difference was observed in tau phosphorylation at these amino acids, nor was the expression of total tau and non-phosphorylated tau proteins different between the ICV-saline and ICV-STZ groups (Fig. 2a-c). Moreover, a comparison of the ICVsaline groups found that tau phosphorylation at Thr205 and Ser396 was reduced in the $\mathrm{Mif}^{-1-}$ mice (Fig. 2a-c). In cerebral cortex, increased tau phosphorylation was also observed at Thr205 and Ser396 in the WT mice receiving ICV-STZ, but tau phosphorylation at these sites was significantly reduced in $\mathrm{Mif}^{-1-}$ mice receiving ICV-saline or ICV-STZ (Additional file 1: Figure S1). These findings suggest that MIF is involved in basal (saline) and induced (STZ) phosphorylation of tau in the ICV mouse model.

In addition to immunoblotting, immunofluorescence staining was performed to confirm increased tau phosphorylation at Thr205 and Ser396 and their distribution 

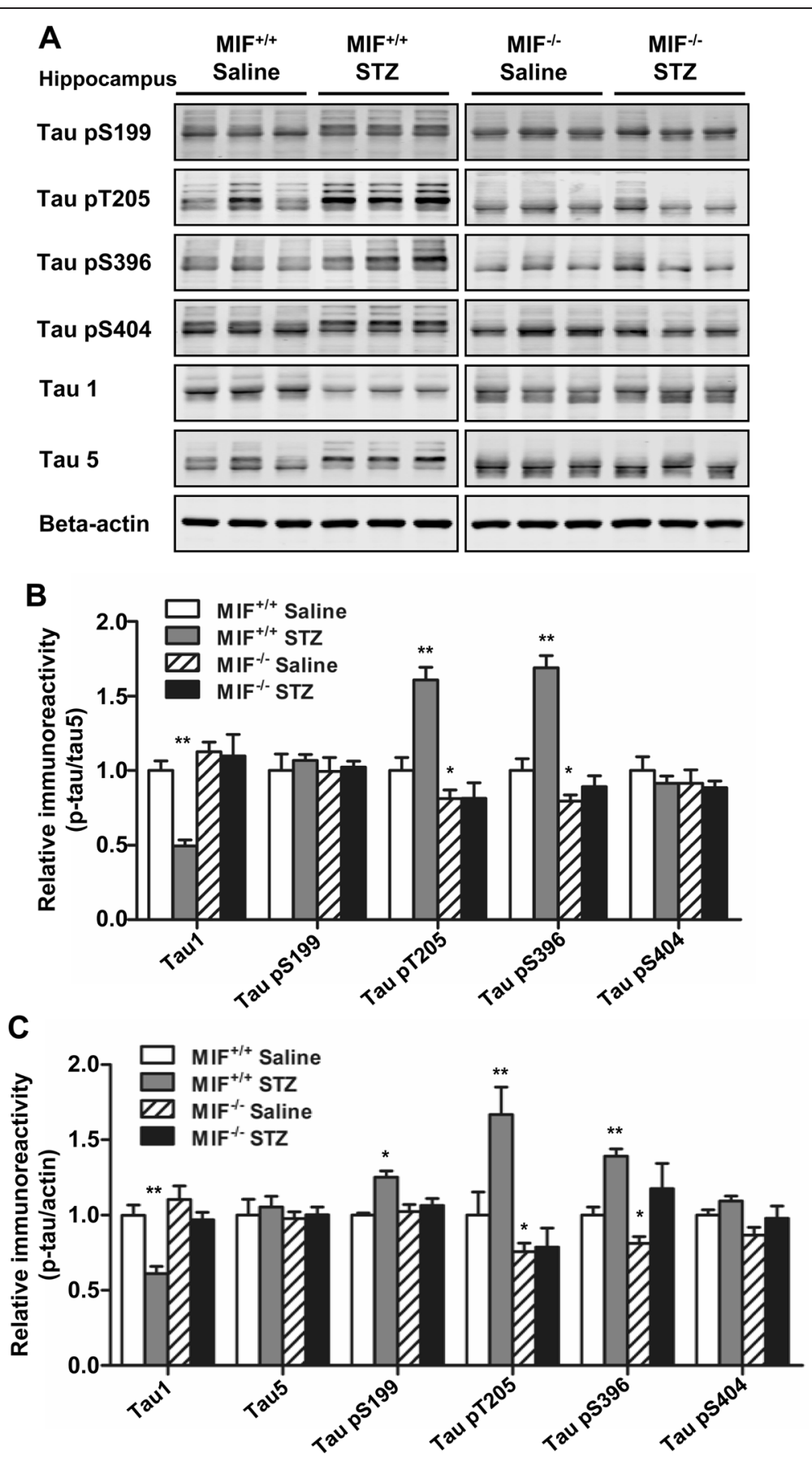

Fig. 2 Effect of MIF on tau hyperphosphorylation in the hippocampus. a Representative Western blots showing tau hyperphosphorylation at S199, T205, S396, and S404 in the hippocampi of WT and Mif ${ }^{-1}$ mice receiving ICV injection of STZ or saline. The levels of non-phosphorylated tau (Tau1) and total tau (Tau5) were also measured. Quantification of the immunoreactivity of representative Western blots, normalized against total tau (b) and $\beta$-actin (c). ${ }^{*} p<0.05,{ }^{* *} p<0.01$ compared with WT mice receiving saline

in the brain. Frozen brain slices were stained with primary anti-Tau (pT205) and anti-Tau (pS396) antibodies, and then treated with Alexa Fluor 568 conjugated anti-rabbit IgG (red). As shown in Fig. 3, increased red fluorescence was observed in the CA1, CA3, and dentate gyrus (DG) areas of hippocampus and in the cortex of ICV-STZ WT mice, compared with WT mice receiving ICV-saline. In contrast, no difference was observed between the STZ and saline groups in $\mathrm{Mif}^{\prime-}$ mice. Similar to the results from immunoblotting analysis, absence of MIF lowered the baseline of phosphorylated tau at Thr205 and Ser396 in the brain slices. These results support the notion that 

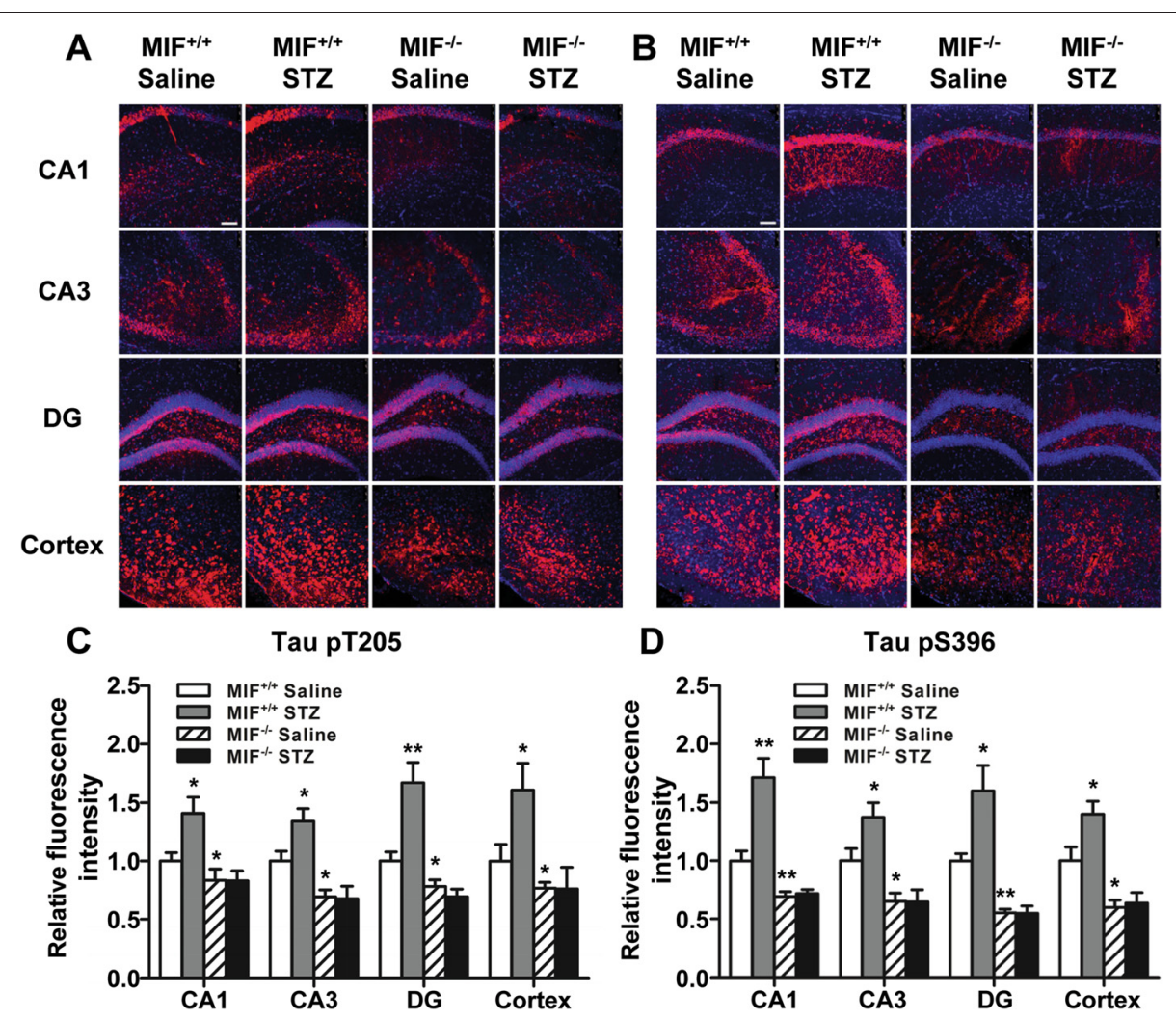

Fig. 3 Staining of phosphorylated tau in brain slices from WT and MIF deficient mice. $\mathbf{a}$, b Frozen slices from cortex and three different regions of hippocampus were stained for phosphorylated tau using rabbit polyclonal anti-Tau (pT205) and anti-Tau (pS396) antibodies. The sections were further treated with Alexa Fluor 568-conjugated anti-rabbit lgG (red fluorescence) and examined under a laser-scanning confocal microscope. Nuclei were stained with DAPI (blue). Scale bar in the upper left panes $75 \mu \mathrm{m}$ ( $\times 200$ magnification). Quantification of the level of tau phosphorylation at pT205 (c) and pS396 (d) in each of the regions, showing differences in fluorescence intensity between WT and Mif ${ }^{-{ }^{-}}$mice receiving STZ and saline. Relative immunofluorescence was measured, and the results are presented as the means \pm SEM based on five viewing fields for each region, using at least two mice. ${ }^{*} p<0.05,{ }^{* *} p<0.01$ compared with $\mathrm{Mif}^{+/+}$mice receiving saline

MIF is involved in regulating tau phosphorylation in the mouse brain.

We also used the APP/PS1 double-transgenic mouse model to confirm MIF involvement in the regulation of tau hyperphosphorylation. APP/PS1 transgenic mice were mated with $\mathrm{Mif}^{/-}$mice. Six-month-old APP/PS1 ${ }^{+/-} \mathrm{Mif}^{\mathrm{f}^{\prime+}}$ mice exhibited significantly increased tau phosphorylation at Ser199, Thr205, Ser396, and Ser404 when compared with age-matched $\mathrm{APP} / \mathrm{PS}^{-/-} \mathrm{Mif}^{+/+}$control mice (Additional file 1: Figure S2). When mated with the $\mathrm{Mif}^{-1-}$ mice to generate $\mathrm{APP} / \mathrm{PSI}^{+/-} \mathrm{Mif}^{-1}$ offspring, tau phosphorylation at these sites was markedly attenuated in comparison with the $\mathrm{APP} / \mathrm{PS}^{+/-} \mathrm{Mif}^{+/+}$mice (Additional file 1: Figure S2).

\section{MIF deficiency attenuates astrocyte activation in mice receiving ICV injection of STZ}

MIF is a proinflammatory cytokine that plays an important role in inflammatory response by stimulating glial cells. It is postulated that MIF-activated glial cells may contribute to tau phosphorylation in neurons. To test this possibility, we first examined the expression of MIF in WT mice that received ICV injection of saline or STZ. Immunofluorescence staining of serial slices from WT mouse brain with antibodies against MIF (green fluorescence) identified a significant upregulation of MIF in CA and DG areas of hippocampus in ICV-STZ mice compared to mice receiving saline (Fig. 4a, b). Moreover, it is evident that the increased MIF colocalized with neurons based on the staining pattern (Fig. 4a), consistent with a previous study [13]. To investigate the activation of astrocytes and their distribution, we stained frozen slices of brain tissue with $\mathrm{CY} 3^{\mathrm{TM}}$ conjugated mouse monoclonal anti-glial fibrillary acidic protein (GFAP; red fluorescence). The expression of GFAP, a protein abundant in activated astrocytes, was increased in ICV-STZ mice. Double immunofluorescence staining showed an increase in MIF colocalization with activated astrocytes in the brain of ICV-STZ mice (Fig. 4a), which showed increased GFAP expression (Fig. 4a, c). These results suggest that ICV injection of STZ induce the expression of MIF and the activation of astrocytes. 

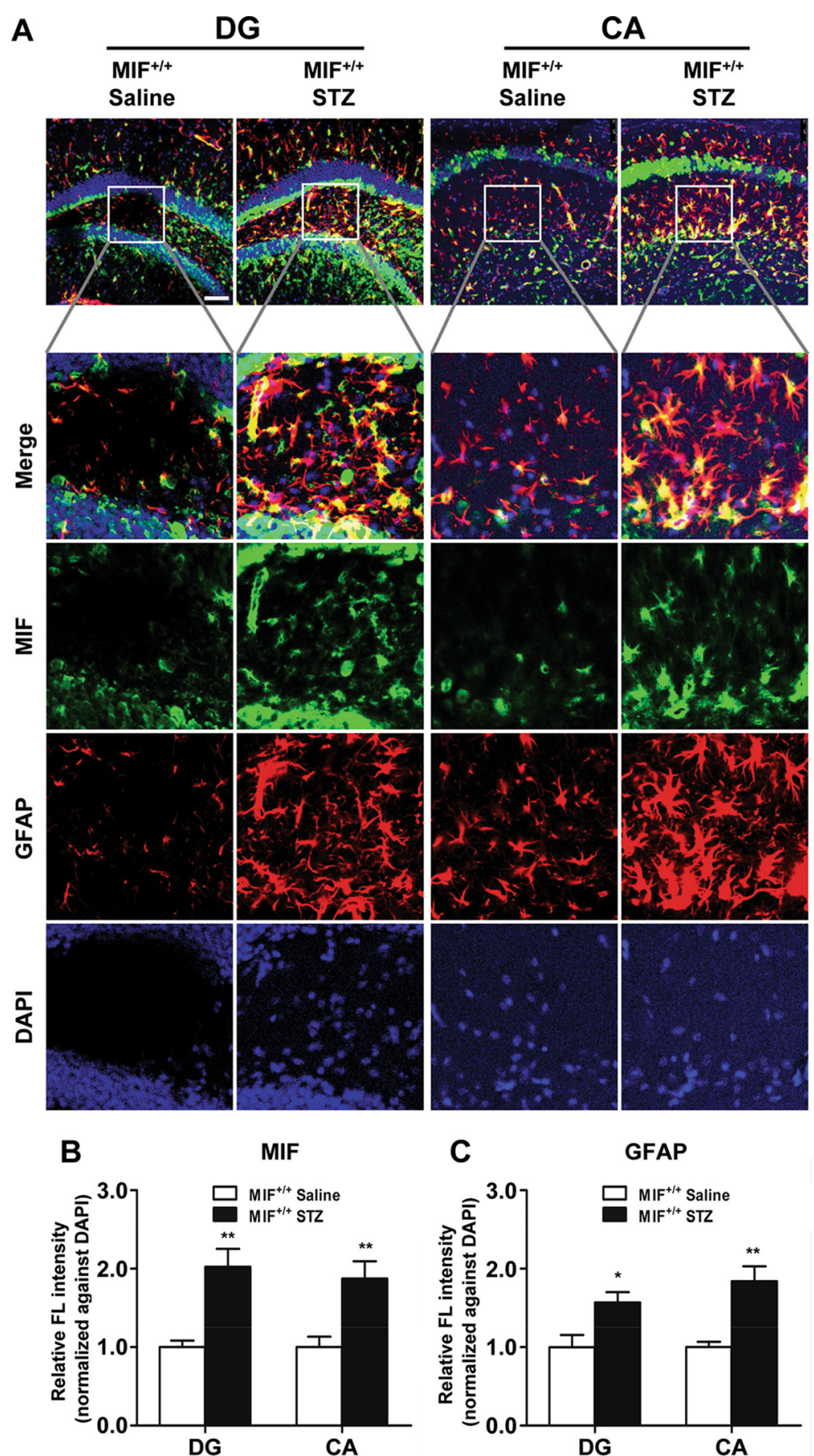

Fig. 4 Upregulation of MIF in ICV-STZ mice and colocalization with GFAP in the hippocampus. a Serial sections of WT mouse brain were stained for MIF protein using a rabbit anti-MIF polyclonal antibody and Alexa Fluor 488-conjugated anti-rabbit lgG (green fluorescence). The sections were subsequently stained for GFAP using a monoclonal anti-GFAP Cy ${ }^{\text {TM }}$ antibody (red fluorescence). Nuclei were stained with DAPI (blue). Images with combined fluorescent channels are shown at $\times 200$ (scale bar in the upper left panel $75 \mu \mathrm{m}$ ). Selected areas are enlarged by 10 times and shown as combined as well as individual fluorescence stains. Quantification of the MIF (b) and GFAP (c) fluorescence was shown after normalization of the green (MIF) and red (GFAP) fluorescence against the blue fluorescence (nuclei). Data shown are means \pm SEM based on multiple experiments $(n=3) .{ }^{*} p<0.05,{ }^{* *} p<0.01$ compared with WT mice receiving saline

To further determine whether MIF is involve in the activation of astroyctes in the $\mathrm{AD}$ model, we compared WT mice with $\mathrm{Mif}^{1-}$ mice that received ICV injection of saline or STZ for the expression of GFAP. Frozen brain slices from these mice were stained with the same anti-GFAP antibody. The results showed a marked increase in GFAP 
stain (red fluorescence) in the CA1, CA3, and DG areas of hippocampus and the cortex of WT mice that received ICV-STZ, compared to the ICV-saline group (Additional file 1: Figure S3A, S3B). In the $\mathrm{Mif}^{-1}$ mice, however, there was no significant change in GFAP fluorescence between the ICV-STZ and ICV-saline groups. These results suggest that MIF is required for the activation of astrocytes in the ICV-STZ mouse model.

\section{MIF regulates tau phosphorylation through activation of astrocytes}

To determine whether MIF acts on neurons directly, we examined the levels of tau phosphorylation in cultured neurons that were exposed to MIF and the MIF inhibitor ISO-1. None of these treatments had a significant effect on tau phosphorylation (Additional file 1: Figure S4A and S4B), suggesting that MIF might affect tau phosphorylation through an indirect mechanism. Since MIF is known to stimulate astrocyte activation, it was postulated that MIF regulation of tau phosphorylation might be mediated through activated astrocytes. Given that STZ injection led to astrocyte activation as well as an increase in glucose level [24], we sought to expose cultured primary astrocytes from WT and $\mathrm{Mif}^{-1}$ newborn mice to high glucose $(75 \mathrm{mM}$ and $150 \mathrm{mM}$ ) to mimic the effect of STZ injection in mice. High glucose conditions have been used in previous studies at glucose concentrations up to $150 \mathrm{mM}$, but these high concentrations are suitable only for cultured cells [24, 27-29]. The level of GFAP in cultured astrocytes was determined by Western blotting and used as an indication of astrocyte activation. As shown in Fig. 5a, the expression level of GFAP in WT astrocytes was significantly increased after 24 and $48 \mathrm{~h}$ of high glucose treatment. However, this effect was absent in $\mathrm{Mif}^{-1}$ astrocytes (Fig. 5a, shaded bars), suggesting that MIF plays a role in high glucose-induced activation of astrocytes.

High glucose treatment of neurons did not alter the level of tau phosphorylation significantly (Additional file 1: Figure S4C), nor did it significantly alter the viability of the cultured neurons and astrocytes (data not shown). To further examine the role of astrocytes in MIFinduced tau hyperphosphorylation, primary neurons from WT and $\mathrm{Mif}^{/-}$mice were cultured in the presence of $\mathrm{CM}$ collected from WT and $\mathrm{Mif}^{/-}$astrocytes exposed to high glucose. Under this experimental condition, primary neurons cultured with the CM from WT astrocytes showed elevated tau phosphorylation (Fig. 5b), whereas those cultured with $\mathrm{CM}$ from $\mathrm{Mif}^{-1}$ astrocytes did not (Fig. 5c). Mif deficiency in neurons had no effect in the level of tau phosphorylation induced by the $\mathrm{CM}$ from high glucoseactivated astrocytes (Fig. 5b, c).

The role for MIF in high glucose-induced astrocyte activation was further tested using the MIF inhibitor
ISO-1. When primary astrocytes were exposed to high glucose in the presence of ISO-1, CM collected from the cultured astrocytes could no longer stimulate tau phosphorylation at T205 in primary cultures of neurons (Fig. 6). Therefore, inhibition of MIF in astrocytes during treatment with high glucose abrogated the ability of the CM to stimulate tau phosphorylation. Collectively, these results suggest that MIF in astrocytes but not neurons plays an important role in elevated tau phosphorylation, which involves mediators released by the activated astrocytes.

\section{Discussion}

The majority of published studies showed that intracellular NFTs often accompany activated astrocytes [30], suggesting possible links between activated astrocytes and tau phosphorylation. In one study, it was reported that astrocytes play an important role for $A \beta$-induced tau phosphorylation in primary neurons [31]. Another study showed that a small molecule, p75NTR ligand (LM11A-31), could attenuate tau hyperphosphorylation and activation of astrocytes in APP transgenic mice [32]. However, there has been no direct evidence for a role of astrocyte activation in tau phosphorylation. As a result, the identity of a suspected mediator remains obscure. The present study documents the effect of MIF in activating astrocytes and modulating tau phosphorylation, thereby providing novel insight into an indirect activation mechanism.

In this study, we evaluated the effects of MIF on tau hyperphosphorylation using two mouse models of AD. ICV injection of STZ is one of the well-known experimental models for sporadic AD [33]. We found that ICV-STZ injection into WT mice induced tau hyperphosphorylation at several AD-related sites including Ser199, Thr205, and Ser396 in the hippocampus and cerebral cortex. This result is consistent with two published studies that compared the ICV-STZ approach with other mouse models of AD [24, 34]. In Mif ${ }^{1-}$ mice, no significant difference was found in the levels of tau phosphorylation between the ICV-STZ and ICV-saline groups. This finding provides first evidence for a potential role of MIF in STZ-induced tau phosphorylation. An involvement of MIF in maintaining the level of tau phosphorylation is also suggested by the observation that the baseline of phosphorylated tau at two of the AD-related sites (Thr205 and Ser396) was reduced in $\mathrm{Mif}^{-1-}$ mice.

Results obtained from another mouse model also support a potential role for MIF in regulating tau phosphorylation. The APP/PS1 transgenic mice have been widely used in studies of AD. Mutations in the genes of amyloid precursor protein (APP) and presenilins (PS1, PS2) increase the production of $A \beta$ and cause familial Alzheimer's disease. The APP/PS1 transgenic mice were mated with the $\mathrm{Mif}^{-1-}$ mice to generate 

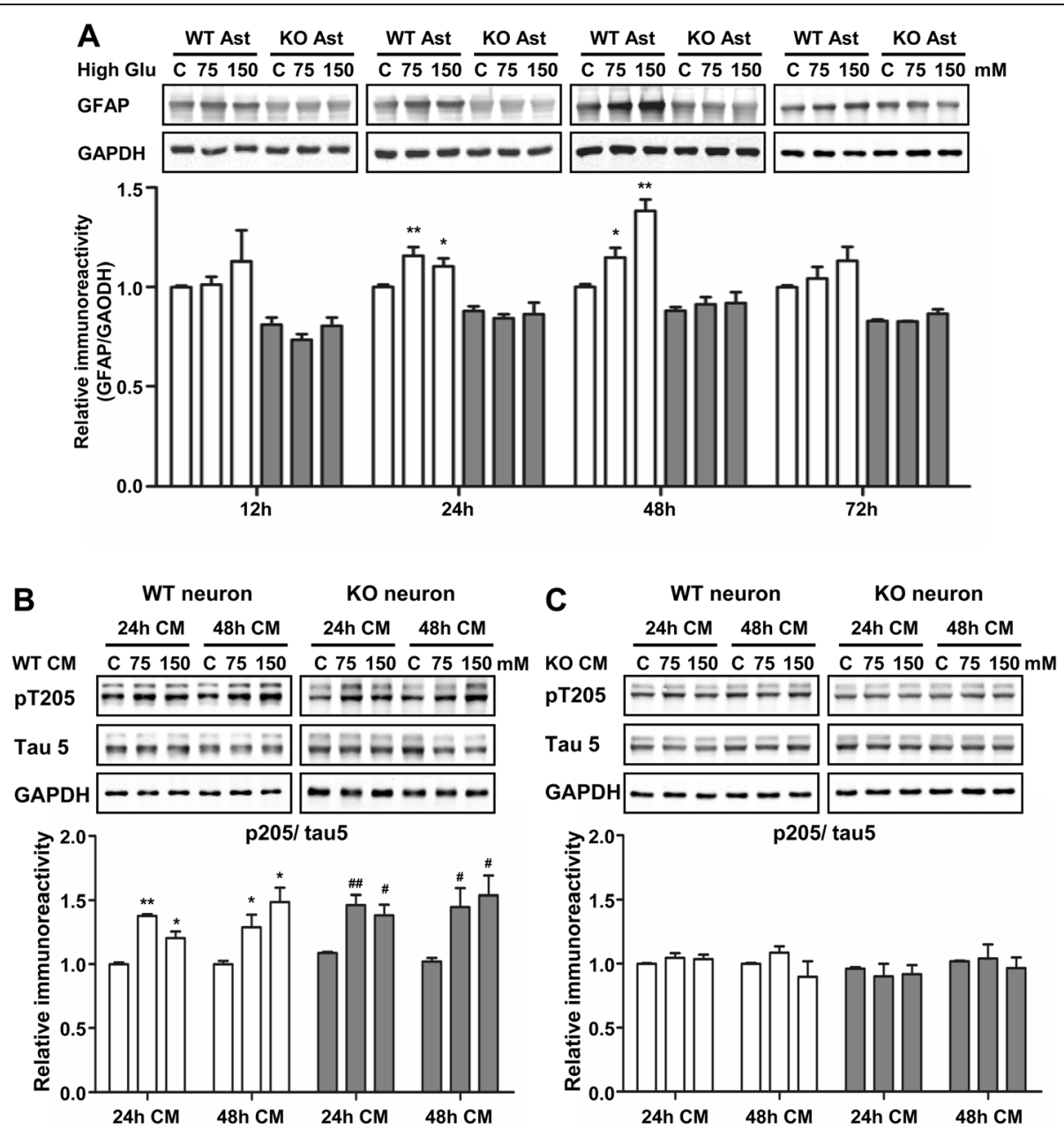

Fig. $5 \mathrm{MIF}$ regulation of tau phosphorylation through activated astrocytes. a Primary cultures of astrocytes (Ast) from WT and Mif ${ }^{-1-}$ newborn mice were treated with DMEM with or without high glucose $(75 \mathrm{mM}$ and $150 \mathrm{mM})$ for 12, 24, 48, and $72 \mathrm{~h}$. The cell lysate was analyzed by Western blotting for the expression of GFAP. The data are presented as densitometric values after normalization against the GAPDH level and are shown as the means \pm SEM from at least three separate experiments; ${ }^{*} p<0.05,{ }^{* *} p<0.01$ scompared with cells without stimulation. For $\mathbf{b}$ and $\mathbf{c}$, freshly isolated neurons from WT and $\mathrm{Mif}^{-1-}$ mice were cultured for $48 \mathrm{~h}$ with conditional medium (CM) of WT (b) and $\mathrm{Mif}^{-1-}$ (c) astrocytes that were exposed to high glucose $(75 \mathrm{mM}$ and $150 \mathrm{mM}$ ) or normal medium (c, DMEM) for 24 and $48 \mathrm{~h}$. Western blotting was performed to detect phosphorylated tau (pT205) in primary neurons. The blots were quantified by densitometry after normalization against total tau (Tau5). All data shown are means \pm SEM of multiple experiments $(n \geq 3)$, each in dulplicate. $\mathbf{c}$ Control. ${ }^{*}$ and ${ }^{\#} p<0.05,{ }^{* *}$ and ${ }^{\# \#} p<0.01$ compared with medium without high glucose

$\mathrm{APP} / \mathrm{PS} 1 / \mathrm{Mif}^{-1-}$ mice for investigation of the potential role of MIF in tau phosphorylation. In these mice, an elevation in the level of phosphorylated tau, typically seen in the APP/PS1 mice, was attenuated in the absence of Mif. This result provides additional evidence for a role of MIF in tau hyperphosphorylation in the AD mouse. The mouse strain may be useful for further studies that will focus on the role of MIF on $A \beta$ plaque formation and $\mathrm{AD}$ progression.

Different brain cells were analyzed for their involvement in MIF regulation of tau phosphorylation. No difference was found in the level of tau phosphorylation in primary cultures of neurons from either WT or $\mathrm{Mif}^{-1-}$ mice. Additionally, neither MIF nor the MIF inhibitor ISO-1 directly affected tau phosphorylation at T205 in primary neurons. Altogether, these results suggest that MIF indirectly regulates tau phosphorylation through other types of cells. Our finding that MIF increases GFAP expression suggests that MIF activates astrocytes. Based on this observation, we postulated that MIF regulates tau phosphorylation through activation of astrocytes. Our experimental results from transfer of astrocyte-conditioned medium to cultured neurons suggest that the MIF-activated astrocytes release mediator(s) that stimulate tau hyperphosphorylation in primary neurons. At present, the nature of the mediator(s) in the MIF-stimulated astrocyte medium remains unclear. Potential candidates include nitric oxide and $\mathrm{S} 100 \mathrm{~B}$ that are known to be released from astrocytes 

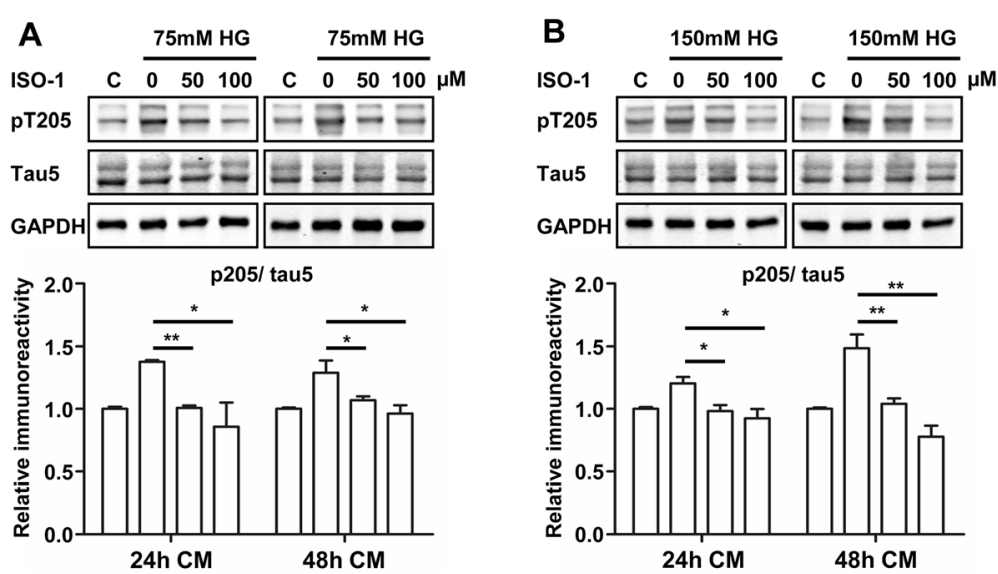

Fig. 6 Inhibition of MIF during high glucose treatment of primary astrocytes abrogated the ability of the CM to stimulate tau phosphorylation in neurons. Primary astrocytes from WT mice were treated with $75 \mathrm{mM}$ (a) or $150 \mathrm{mM}$ (b) of high glucose in the presence or absence of the MIF inhibitor ISO-1 (50 $\mu \mathrm{M}$ and $100 \mu \mathrm{M})$. CM collected after $24 \mathrm{~h}$ and $48 \mathrm{~h}$ was added to primary cultures of mouse neurons. After $48 \mathrm{~h}$, the neurons were harvested and the level of tau phosphorylation (pT205) was determined by Western blotting. Densitometry readings of the blots were normalization against those of total tau (Tau5). The level of tau phosphorylation in the untreated samples (DMEM without high glucose or ISO-1; marked as C in the first lane) was set at 1.0, with which other samples were compared. Data presented in bar charts are means \pm SEM of three individual experiments. ${ }^{*} p<0.05,{ }^{*} p<0.01 \mathrm{vs}$. high glucose medium without ISO-1

and induce tau hyperphosphorylation in cell culturebased experiments [5,35]. Future studies will focus on these mediator(s) for their involvement in MIF-stimulated tau hyperphosphorylation through astrocytes.

\section{Conclusions}

MIF is an inflammatory cytokine with pleiotropic functions in many chronic inflammatory diseases. Using two mouse models of $\mathrm{AD}$, we demonstrate that MIF plays a role in the regulation of tau hyperphosphorylation in neurons. MIF does not directly affect tau phosphorylation; instead, MIF activates astrocytes that release mediator(s) capable of stimulating tau hyperphosphorylation in neurons. Our findings corroborate the notion that MIF may be a potential target for therapeutic intervention of AD.

\section{Additional file}

Additional file 1: Figure S1. Involvement of MIF in tau

hyperphosphorylation in the cerebral cortex of mouse brain. Figure S2. MIF regulation of tau hyperphosphorylation in APP/PS1 double-transgenic mouse model. Figure S3. Effect of MIF on astrocyte activation in mouse brain. Figure S4. Absence of direct effect of MIF, ISO-1, and high glucose on primary neurons. (DOCX $1588 \mathrm{~kb})$

\section{Abbreviations \\ AD: Alzheimer's disease; APP: amyloid precursor protein; AB: amyloid beta; CM: conditioned medium; CSF: cerebral spinal fluid; DAPI: 4,6-Diamidino-2- phenylindole; DMEM: Dulbecco's modified Eagle's medium; GFAP: glial fibrillary acidic protein; ICV: intracerebroventricular; MIF: macrophage migration inhibitory factor; NFTs: neurofibrillary tangles; PS1: presenilin-1; STZ: streptozotocin; TBS: Tris-buffered saline; WT: wild type.}

\section{Competing interests}

The authors declare that they have no competing interests.

\section{Authors' contributions}

$S Q L, J Z H, D W$, and JL carried out the in vivo and in vitro studies. SQL and YY processed and analyzed the data. FQ, GHF, and RB provided the critical reagents and participated in the discussions. YY and RDY designed the study and wrote the manuscript. All authors have read and agree with the contents of the manuscript.

\section{Acknowledgements}

This work was supported by grants from National Natural Science Foundation of China (Grant 31270941 to R.D.Y. and Grant 81200843 to Y.Y.), from National Basic Research Program of China (973 Program, Grant 2012CB518001, to R.D.Y.), from the Specialized Research Fund for the Doctoral Program of Higher Education of China (Grant 20120073110069, to R.D.Y. and Grant 20120073120091, to Y.Y.), and from National Institutes of Health (R01Al042310, to R.B.).

\section{Author details}

'School of Pharmacy, Shanghai Jiao Tong University, Shanghai 200240, China. ${ }^{2} \mathrm{GlaxoSmithKline} \mathrm{Research} \mathrm{and} \mathrm{Development} \mathrm{Center,} \mathrm{Shanghai}$ 201203, China. ${ }^{3}$ Department of Medicine, Yale University School of Medicine, New Haven, CT 06520, USA. ${ }^{4}$ Institute of Chinese Medical Sciences, University of Macau, Macau, SAR, China.

Received: 9 April 2015 Accepted: 9 September 2015 Published online: 17 September 2015

\section{References}

1. Birch AM, Katsouri L, Sastre M. Modulation of inflammation in transgenic models of Alzheimer's disease. J Neuroinflammation. 2014;11:25.

2. Allaman I, Belanger M, Magistretti PJ. Astrocyte-neuron metabolic relationships: for better and for worse. Trends Neurosci. 2011;34:76-87.

3. Li Y, Liu L, Barger SW, Griffin WS. Interleukin-1 mediates pathological effects of microglia on tau phosphorylation and on synaptophysin synthesis in cortical neurons through a p38-MAPK pathway. J Neurosci. 2003;23:1605-11.

4. Quintanilla RA, Orellana DI, Gonzalez-Billault C, Maccioni RB. Interleukin-6 induces Alzheimer-type phosphorylation of tau protein by deregulating the cdk5/p35 pathway. Exp Cell Res. 2004;295:245-57.

5. Saez TE, Pehar M, Vargas M, Barbeito L, Maccioni RB. Astrocytic nitric oxide triggers tau hyperphosphorylation in hippocampal neurons. In Vivo. 2004;18:275-80.

6. Metz CN, Bucala R. Role of macrophage migration inhibitory factor in the regulation of the immune response. Adv Immunol. 1997;66:197-223. 
7. Matsunaga J, Sinha D, Pannell L, Santis C, Solano F, Wistow GJ, et al. Enzyme activity of macrophage migration inhibitory factor toward oxidized catecholamines. J Biol Chem. 1999;274:3268-71.

8. Morand EF, Leech M, Bernhagen J. MIF: a new cytokine link between rheumatoid arthritis and atherosclerosis. Nat Rev Drug Discov. 2006:5:399-410.

9. Calandra T, Bernhagen J, Metz CN, Spiegel LA, Bacher M, Donnelly T, et al. MIF as a glucocorticoid-induced modulator of cytokine production. Nature. 1995;377:68-71.

10. Calandra T, Roger T. Macrophage migration inhibitory factor: a regulator of innate immunity. Nat Rev Immunol. 2003;3:791-800.

11. Bach JP, Deuster O, Balzer-Geldsetzer M, Meyer B, Dodel R, Bacher M. The role of macrophage inhibitory factor in tumorigenesis and central nervous system tumors. Cancer. 2009;115:2031-40.

12. Bryan KJ, Zhu X, Harris PL, Perry G, Castellani RJ, Smith MA, et al. Expression of CD74 is increased in neurofibrillary tangles in Alzheimer's disease. Mol Neurodegener. 2008;3:13.

13. Bacher M, Meinhardt A, Lan HY, Dhabhar FS, Mu W, Metz CN, et al. MIF expression in the rat brain: implications for neuronal function. Mol Med. 1998;4:217-30

14. Popp J, Bacher M, Kolsch H, Noelker C, Deuster O, Dodel R, et al. Macrophage migration inhibitory factor in mild cognitive impairment and Alzheimer's disease. J Psychiatr Res. 2009:43:749-53.

15. Yoshiyama Y, Arai K, Oki T, Hattori T. Expression of invariant chain and pro-cathepsin L in Alzheimer's brain. Neurosci Lett. 2000;290:125-8.

16. Bacher M, Deuster O, Aljabari B, Egensperger R, Neff F, Jessen F, et al. The role of macrophage migration inhibitory factor in Alzheimer's disease. Mol Med. 2010;16:116-21.

17. Al-Abed Y, VanPatten S. MIF as a disease target: ISO-1 as a proof-of-concept therapeutic. Future Med Chem. 2011;3:45-63.

18. Gong CX, labal K. Hyperphosphorylation of microtubule-associated protein tau: a promising therapeutic target for Alzheimer disease. Curr Med Chem. 2008;15:2321-8.

19. Fingerle-Rowson G, Petrenko O, Metz CN, Forsthuber TG, Mitchell R, Huss R, et al. The p53-dependent effects of macrophage migration inhibitory factor revealed by gene targeting. Proc Natl Acad Sci U S A. 2003;100:9354-9.

20. Chen Y, Liang Z, Tian Z, Blanchard J, Dai CL, Chalbot S, et al. Intracerebroventricular streptozotocin exacerbates Alzheimer-like changes of 3xTg-AD mice. Mol Neurobiol. 2014;49:547-62.

21. Yu Y, Wang JR, Sun PH, Guo Y, Zhang ZJ, Jin GZ, et al. Neuroprotective effects of atypical D1 receptor agonist SKF83959 are mediated via D1 receptor-dependent inhibition of glycogen synthase kinase-3 beta and a receptor-independent anti-oxidative action. J Neurochem. 2008;104:946-56.

22. Peng L, Yu Y, Liu J, Li S, He H, Cheng N, et al. The chemerin receptor CMKLR1 is a functional receptor for amyloid-beta peptide. J Alzheimers Dis. 2015;43:227-42.

23. Yu Y, Liu J, Li SQ, Peng L, Ye RD. Serum amyloid A differentially activates microglia and astrocytes via the PI3K pathway. J Alzheimers Dis. 2014;38:133-44.

24. Grunblatt E, Salkovic-Petrisic M, Osmanovic J, Riederer P, Hoyer S. Brain insulin system dysfunction in streptozotocin intracerebroventricularly treated rats generates hyperphosphorylated tau protein. J Neurochem. 2007;101:757-70

25. Salkovic-Petrisic M, Knezovic A, Hoyer S, Riederer P. What have we learned from the streptozotocin-induced animal model of sporadic Alzheimer's disease, about the therapeutic strategies in Alzheimer's research. J Neura Transm. 2013;120:233-52

26. Pathan AR, Viswanad B, Sonkusare SK, Ramarao P. Chronic administration of pioglitazone attenuates intracerebroventricular streptozotocin inducedmemory impairment in rats. Life Sci. 2006;79:2209-16.

27. Gewolb $1 \mathrm{H}, \mathrm{O}$ 'Brien J, Palese TA, Phillip M. High glucose and insulin decrease fetal lung insulin receptor mRNA and tyrosine kinase activity in vitro. Biochem Biophys Res Commun. 1994;202:694-700.

28. Cheng JT, Liu IM, Chi TC, Tzeng TF, Lu FH, Chang CJ. Plasma glucoselowering effect of tramadol in streptozotocin-induced diabetic rats. Diabetes. 2001;50:2815-21.

29. Yan J, Zhang Z, Shi H. HIF-1 is involved in high glucose-induced paracellular permeability of brain endothelial cells. Cell Mol Life Sci. 2012;69:115-28.

30. Meraz-Rios MA, Toral-Rios D, Franco-Bocanegra D, Villeda-Hernandez J, Campos-Pena V. Inflammatory process in Alzheimer's disease. Front Integr Neurosci. 2013;7:59.
31. Garwood CJ, Pooler AM, Atherton J, Hanger DP, Noble W. Astrocytes are important mediators of Abeta-induced neurotoxicity and tau phosphorylation in primary culture. Cell Death Dis. 2011;2:e167.

32. Nguyen TV, Shen L, Vander Griend L, Quach LN, Belichenko NP, Saw N, et al. Small molecule p75NTR ligands reduce pathological phosphorylation and misfolding of tau, inflammatory changes, cholinergic degeneration, and cognitive deficits in AbetaPP $(L / S)$ transgenic mice. J Alzheimers Dis. 2014;42:459-83.

33. Mehla J, Pahuja M, Gupta YK. Streptozotocin-induced sporadic Alzheimer's disease: selection of appropriate dose. J Alzheimers Dis. 2013;33:17-21.

34. Chen $Y$, Liang Z, Blanchard J, Dai CL, Sun S, Lee MH, et al. A non-transgenic mouse model (icv-STZ mouse) of Alzheimer's disease: similarities to and differences from the transgenic model (3xTg-AD mouse). Mol Neurobiol. 2013;47:711-25.

35. Esposito G, Scuderi C, Lu J, Savani C, De Filippis D, luvone T, et al. S100B induces tau protein hyperphosphorylation via Dickopff-1 up-regulation and disrupts the Wnt pathway in human neural stem cells. J Cell Mol Med. 2008;12:914-27.

\section{Submit your next manuscript to BioMed Central and take full advantage of:}

- Convenient online submission

- Thorough peer review

- No space constraints or color figure charges

- Immediate publication on acceptance

- Inclusion in PubMed, CAS, Scopus and Google Scholar

- Research which is freely available for redistribution 